\title{
QUADERNI Quaderni
}

Communication, technologies, pouvoir

99-100 | Hiver 2019-2020

Technologies et jeux de pouvoir

\section{Arnaud Mercier, Nathalie Pignard-Cheynel , \#info, Commenter et partager l'actualité sur Twitter et Facebook}

\section{Christine Barats}

\section{OpenEdition}

\section{Journals}

Édition électronique

URL : https://journals.openedition.org/quaderni/1581

DOI : 10.4000/quaderni.1581

ISSN : 2105-2956

Éditeur

Les éditions de la Maison des sciences de l'Homme

\section{Édition imprimée}

Date de publication : 5 janvier 2020

Pagination : 159-167

\section{Référence électronique}

Christine Barats, «Arnaud Mercier, Nathalie Pignard-Cheynel ,\#info, Commenter et partager l'actualité sur Twitter et Facebook », Quaderni [En ligne], 99-100 | Hiver 2019-2020, mis en ligne le 05 janvier 2020, consulté le 05 janvier 2023. URL : http://journals.openedition.org/quaderni/1581 ; DOI : https:// doi.org/10.4000/quaderni.1581 


\section{Compte rendu}

\section{\#info, Commenter et partager l'actualité sur Twitter et Facebook}

\section{Arnaud Mercier, Nathalie Pignard-Cheynel (dir.)}

Éditions Fondation Maison des Sciences de l'Homme, Paris, 2018, 352 pages

par Christine Barats

Université Paris Descartes,

Cerlis, UMR 8070

Les transformations des façons de s'informer en contexte numérique, tout autant que la place des smartphones comme terminal de connexion soulignent l'actualité de cet ouvrage dirigé par Arnaud Mercier, professeur en sciences de l'information et de la communication (SIC) à l'université Paris 2 Panthéon Assas, et Nathalie Pignard-Cheynel, professeure assistante en journalisme numérique à l'université de Neuchâtel. Il s'inscrit dans le prolongement de travaux portant sur les transformations du journalisme à l'ère des réseaux socio-numériques (RSN) et rassemble 10 contributions de 12 auteurs spécialisés en sociologie du journalisme et des médias, en sciences de l'information et de la communication et en sciences du langage. Il est le fruit d'un programme de recherche financé par l'ANR (2014-2017), intitulé «Info-RSN ».

La perspective retenue consiste à examiner la place et le rôle des RSN dans la circulation de l'information. Partant du constat que l'information journalistique se voit, se lit et s'écoute de plus en plus sur les «murs » des réseaux sociaux, l'ouvrage s'attache à observer et à analyser la façon dont les informations sont partagées sur Twitter ou commentées sur Facebook. Il prête attention aux spécificités techniques de ces dispositifs notamment aux modalités sémio-discursives de cette circulation, ce qui constitue un des points forts de l'ouvrage. De nombreuses illustrations en couleur rendent compte de la richesse des données collectées et analysées, ainsi que de la diversité des approches (principalement statistique textuelle, analyse sémio-discursive, enquête par questionnaire, entretiens et ethnographie en ligne).

Le parti-pris partagé par les auteurs est d'examiner la façon dont circulent 
les informations, non pas du point de vue des rédactions ${ }^{1}$ - objet d'une précédente recherche sur les modalités d'appropriation de ces dispositifs par les journalistes, menée en 2012 par certains membres de ce collectif ${ }^{2}$-, mais du point de vue de leur appropriation, c'est-à-dire quelles sont les modalités de partage et de commentaire chez leurs publics. Les contributions s'adossent aux données relatives à la circulation d'actualités publiées par 31 sites de médias (quotidiens nationaux, hebdomadaires, mensuels, pureplayers, TV et radio $^{3}$ ) sur Twitter et Facebook, ainsi que des données complémentaires, fruits de recherches menées par des membres de ce collectif (ethnographie en ligne menée sur Facebook, corpus de tweets politiques...).

Fruit d'une recherche interdisciplinaire, la publication témoigne, d'une manière générale, d'une collaboration réussie entre informaticiens et chercheurs en SHS. Le volume des données (plus de 38 millions de tweets collectés entre novembre 2015 et mars 2016) et leur hétérogénéité (signes alphanumériques, images, vidéos, nombre de followers, de retweets...) ont nécessité le recours à une base de données facilitant la constitution de corpus ad hoc et montrent l'intérêt de mobiliser des outils de traitement automatique en particulier pour l'analyse des données textuelles, en l'occurrence Iramuteq ${ }^{4}$, à la base de nombreux résultats. Ajoutons que les perspectives de recherche, ainsi que le choix des données analysées attestent d'un souci de ne pas se limiter à certains comptes Twitter, en particulier humains, mais d'examiner la circulation d'actualités citant ces 31 médias, y compris dans le cas de comptes Twitter animés par des robots qui retweetent de manière automatique certaines informations comme le montre le chapitre 1 (Nathalie Pignard-Cheynel et Dario Compagno). L'identification de ces comptes témoigne de l'intérêt des auteurs pour la technicisation de certaines pratiques de circulation, tout autant que pour les caractéristiques techno-sémiotiques de cette circulation. Certains chapitres examinent ainsi de manière approfondie la place de l'arobase dans les « discours d'escorte » sur Twitter (chapitre 2), les caractéristiques des hashtags dans le partage de l'information (chapitre 3), le rôle des images (chapitre 4) ou celui des émoticônes (chapitre 10). Ces éléments contribuent à la circulation de l'information, à sa mise en visibilité et à sa visée argumentative. Notons également que la présentation et la description des différents protocoles mis en œuvre pour chacun des chapitres (corpus $a d h o c$, enquête par questionnaire...) sont utiles aux lecteurs car cela permet de mettre au jour les choix inhérents à la sélection des données et à la constitution de corpus, et parfois de souligner les limites de leur interprétation

\footnotetext{
1. Une publication récente complète cette perspective : Journalisme mobile, Usages informationnels, stratégies éditoriales et pratiques journalistiques, sous la direction de Nathalie Pignard-Cheynel et Lar van Dievoet, Louvain-La-Neuve, De Boeck Supérieur, 2019, 288 pages.

2. Arnaud Mercier et Nathalie Pignard-Cheynel, "Mutations du journalisme à l'ère du numérique : un état des travaux ", Revue française des sciences de l'information et de la communication, $n^{\circ}$ 5, 2014, en ligne : http://journals. openedition.org/rfsic/1097. Voir également https://obsweb.net/blog/2012/05/14/ enquete-sur-les-usagesdes-reseaux-sociaux-parles-journalistes-francais/

3. Citons pour illustrer lemonde.fr, lefigaro.fr, lequipe.fr, parismatch.fr, lexpress.fr, 20minutes.fr, bfmtv.fr, itele.fr, rue89.com, franceinter.fr, franceinfo. $\mathrm{fr}$, mediapart.fr etc. (liste exhaustive indiquée page 13 de l'ouvrage)
}

4. http://www.iramuteq.org/ 
(manque d'informations sur les profils socio-démographiques des internautes qui partagent le plus d'informations (p. 52), importance du retour au texte dans le cas d'outils de statistique textuelle ou difficultés d'interprétation (p. 160), limites et possibles biais liés au déclaratif dans le cas de l'enquête par questionnaire (p. 190), etc.).

D'un point de vue formel, l'ouvrage est organisé en deux parties. La première partie met l'accent sur les tactiques de partage d'informations (qui partage quoi, comment et avec quelles caractéristiques sémio-discursives) et rassemble six contributions, dont quatre portent sur Twitter et deux sur Facebook. Toutes les contributions de la deuxième partie portent sur Twitter et mettent l'accent sur un des aspects de la mise en circulation d'articles, à savoir leur dimension polémique. Le premier chapitre constitue un arrièreplan nécessaire pour comprendre l'économie globale du partage d'informations sur Twitter. Les auteurs ont ainsi pu examiner la place des robots (bots et newsbots) ainsi que des algorithmes et montrent que ces pratiques de tweets qui visent à amplifier la circulation des informations, y compris par les médias eux-mêmes, ne sont pas négligeables : 22\% des 38 millions de tweets présents dans la base initiale du projet «Info-RSN » ont été mis en circulation par des bots. L'analyse met en exergue une disparité dans les médias partagés : certains médias sont très partagés (lemonde.fr et lefigaro.fr), en lien avec leur productivité quotidienne, et 6 médias s'avèrent les plus mentionnés (lemonde.fr, lefigaro.fr, lequipe.fr, lesechos.fr, le parisien.fr ou l'express.fr), soit $71 \%$ du corpus, soulignant que sur les 31 médias étudiés, 25 occupent une place limitée par rapport à ces 6 médias très cités. Grâce à Iramuteq, les auteurs ont examiné la thématisation des contenus retweetés (p. 44 et suivantes) et ont observé une place conséquente des actualités sportives (34,3\% des tweets), en particulier footballistiques (plus des $2 / 3$ des actualités sportives), confirmant la place de lequipe.fr, en particulier par rapport aux sujets économiques ( $25,2 \%$ des tweets), de politique intérieure (plus de $17 \%$ des tweets) et étrangère $(13,7 \%$ des tweets) ou de technologie $(9,7 \%)$.

Si certaines thématiques suscitent le partage, le deuxième chapitre prête attention aux caractéristiques sémio-discursives de ces partages et analyse les discours qui accompagnent le partage d'une URL sur Twitter, ce que les auteures qualifient de « discours d'escorte » (Chapitre 2, Magali Bigey et Justine Simon). Les auteures mettent ainsi au jour les marques explicites d'incitation au partage (« \#urgent », «\#insolite »), qui relèvent parfois d'un 
simulacre de dialogisme interlocutif et atteste des stratégies communicationnelles des twittos. Les stratégies d'interpellation montrent la diversité et l'importance des tactiques pour faire circuler une information (mentions d'une personne avec l'utilisation de l'arobase, questions souvent rhétoriques, textes courts d'accroche ou d'incitation à l'action...). Compte tenu du parti-pris de l'ouvrage de ne pas se situer du côté des rédactions, les auteures ont exclu de leur analyse les tweets des journalistes. Leur prise en compte permettrait cependant de prolonger ces investigations et de mesurer s'ils adoptent les mêmes tactiques de communication pour inciter au partage. Cette perspective sémio-discursive au regard du discours d'escorte est complétée par le chapitre 4 (Bénédicte Toullec et Justine Simon) qui porte sur la place des images dans la circulation de l'information. Ces deux chapitres proposent ainsi des cadres théoriques intéressants pour aborder la dimension textuelle de la circulation ou bien celle de la multimodalité des tweets (images, iconotexte, films, caricatures, infographies, photomontage...). De nombreux exemples illustrent le chapitre 4 et montrent que la présence d'images relève du « style Twitter » : les médias l'utilisent majoritairement afin de susciter des retweets, tout autant que les twittos. Les auteures mettent au jour la diversité du recours à l'image sur Twitter (photomontage, détournement...) et montrent que les twittos l'emploient dans une perspective subjective d'exercice de la critique : les images fonctionnent comme administration de la preuve, et également comme tactiques argumentatives de nature polémique, car les attaques sont fréquentes sur Twitter. Entre humour, cynisme, ironie et critique, la présence d'images suscite le retweet. Si les cas des mèmes et des gifs ne sont abordés qu'en conclusion, cette contribution rend compte de l'expressivité des images et de leur portée dans le cas d'attaques ad personam destinées à décrédibiliser une personnalité.

Le rôle des hashtags dans les tactiques de partage, c'est-à-dire la portée argumentative des signes graphiques et visuels qui ont forgé le « style Twitter », est l'objet du troisième chapitre (Arnaud Mercier). Puissant outil de coordination, le hashtag est appréhendé dans le prolongement des travaux de Marie-Anne Paveau ${ }^{5}$ comme une production technolangagière, au même titre que le lien hypertextuel, à la fois comme un outil de création d'un fil d'actualité et également comme un outil de mobilisation. La diversité des usages du hashtag au regard du corpus étudié montre la variété de ses appropriations. L'essai de typologie de ces usages - nonobstant les limites inhérentes à tout essai de typologie - indique que le « hashtag indexateur journalistique », très présent

\footnotetext{
5. Voir en particulier le blog : https://technodiscours.hypotheses.org/ ainsi que le dictionnaire : Marie-Anne Paveau, L'analyse du discours numérique. Dictionnaire des formes et des pratiques, Paris, Hermann, 2017.
} 
6. Dominique Pasquier L'Internet des familles modestes. Enquête dans la France rurale, Paris, Presses des Mines, coll. « Sciences sociales », 2018. et très fruste dans le cas des bots (voir chapitre 1), permet de « rubriquer » et de thématiser l'information (\#sport, \#gaza...). Souvent à l'initiative des médias, l'emploi de ce type de hashtag s'apparente à la pratique de presse du rubriquage de l'information et met au jour la transposition et l'adaptation de certaines pratiques journalistiques dans le cas des RSN. Le « hashtag événementiel » permet quant à lui de repérer et éclairer un événement et se distingue du « hashtag grappin » qui relève davantage du cadrage et du label qui suscite l'intérêt dans le cas d'une veille informationnelle. Ce dernier est très utilisé par l'extrême droite ou des groupes conservateurs (\#théoriedugenre, p.107). Il est parfois proche du « hashtag axiologique » qui marque l'expression d'un jugement (\#hypocrite, \#enfin...). Le « hashtag clin d'œil » ou le « hashtag ironique » relève du hashtag ludique identifié par Marie-Anne Paveau et montre le spectre large des appropriations de ce technolangage, tout autant que sa richesse, façonnant ce mot-clic comme mot-argument.

Les chapitres 5 et 6 s'intéressent plus spécifiquement à la circulation de l'information sur Facebook. Le chapitre 5 (Alan Ouakrat, Nathalie PignardCheynel \& Arnaud Mercier) s'adosse à une enquête par questionnaire menée auprès d'étudiants de l'université de Lorraine et de l'université PanthéonAssas, Paris 2, sur leurs pratiques informationnelles sur Facebook. 1820 questionnaires ont été dépouillés et ils ont été complétés par 15 entretiens. Les résultats montrent l'importance de Facebook et des smartphones dans le partage et la circulation de l'information. Les auteurs notent que cet infomédiaire facilite le brouillage des frontières entre information et pratique ludique. Les médias traditionnels occupent une place importante, au regard des données déclaratives collectées, ce qui conforte une forme légitime des pratiques informationnelles, compte tenu de l'intérêt déclaré pour les médias généralistes nationaux. Les médias parodiques sont également très cités, confirmant l'hypothèse selon laquelle Facebook est un lieu de divertissement avant d'être un lieu d'information (p. 180). L'importance de la communication interpersonnelle est soulignée (p. 195), de même que la diversité des pratiques ( $1 / 3$ des enquêtés ne partagent pas d'actualités sur leur mur, p. 185), ainsi que le profil atypique des enquêtés (jeunes étudiants de 18 à 24 ans, avec $62 \%$ de femmes), ce qui limite la généralisation des résultats. Les travaux menés par Dominique Pasquier montrent ainsi des pratiques en ligne différentes en milieu rural ${ }^{6}$. Le chapitre 6 (Coralie Le Caroff) mobilise quant à lui une étude ethnographique menée en ligne en 2014 lors d'une recherche doctorale sur 
le partage d'actualité dans le cas de réseaux personnels sur Facebook, qui a été complétée par 35 entretiens. Les observations confirment la prégnance de la construction de soi en ligne et attestent des enjeux de communication interpersonnelle. Elles mettent également au jour l'hétérogénéité des pratiques. Certains profils sont très actifs, ils sont cependant minoritaires : les très actifs « ego-visibles » (7 enquêtés sur 35$)$ et les actifs « ego-centrés » (9 sur 35) publient et contribuent ainsi à la mise en visibilité et à la circulation d'actualités. Les « partageurs » (29\% des enquêtés) et les « spectateurs » se contentent de lire et ne publient pas. Ils s'avèrent majoritaires. La typologie qui est proposée des différents profils permet de confirmer que les profils très actifs restent minoritaires au regard de ceux qui utilisent Facebook dans une perspective de veille informationnelle et d'échanges interpersonnels. Certains enquêtés évoquent ainsi la dimension conviviale de Facebook, et pour les plus actifs, des pratiques de drague (p. 220).

La deuxième partie met l'accent sur la dimension polémique de la mise en circulation d'articles. Les tactiques propres à Twitter sont ainsi mises au jour dans le cas de la polémique et des attaques sur Twitter contre Christiane Taubira lors de la réforme pénale de juin 2014 (Chapitre 7, Brigitte Sebbah, Romain Badouard \& Arnaud Mercier). Pour examiner les tweets polémiques, les auteurs ont extrait de la base « info RSN » les tweets comprenant le nom « Taubira » entre le $1^{\text {er }}$ juin et le 31 juillet 2014, soit 5772 tweets (p. 232). Après élimination des doublons et des tweets sans discours d'escorte, 1824 tweets ont été examinés afin de mettre au jour leurs traits saillants. Si trois registres d'expression ont été identifiés (humour dont ironie, agressivité et interpellation), les auteurs mettent au jour ce qui caractérise le « style Twitter » : jeux avec la ponctuation, avec la casse des lettres, onomatopées, interjections etc. (p. 233). Ces caractéristiques scripturaires et discursives ne sont pas exclusives à Twitter et pourront intéresser les chercheurs qui examinent les écrits et commentaires en contexte numérique. Les marques d'humour sont très présentes (62\%) ainsi que celles d'un ton agressif (33\%). Les auteurs relèvent sans surprise que le recours aux insultes est une des caractéristiques des tweets agressifs (p. 257) et soulignent en conclusion qu'un nombre non négligeable de tweets d'opinion instrumentalisent un fait d'actualité. C'est une des raisons pour lesquelles, les auteurs privilégient le syntagme « discours d'escorte » plutôt que « commentaire », car cela permet de prendre en compte des « commentaires » qui ne sont pas une réaction à une actualité mais au contraire l'argument de départ auquel vient s'adjoindre 
7. Le chapitre ne précise ni les noms des personnalités, $\mathrm{ni}$ les groupes. Le travail d'annotation des données collectées qui a été opéré sur le corpus grâce au balisage TEI (Text Encoding Initiative) montre l'intérêt de recourir à des outils de traitement automatique. Voir https://hal. archives-ouvertes.fr/hal01270984v2/document à titre d'illustration le lien URL de l'actualité (p. 242).

Le chapitre 8 (Agata Jackiewicz) met également l'accent sur la dimension polémique de Twitter au regard de la controverse autour du mariage, de la procréation et de la filiation issue de la GPA (gestation pour autrui) et de la PMA (procréation médicalement assistée), à partir de l'examen d'une période de 24 heures (5 octobre 2014). Notons rapidement l'intérêt de la prise en compte de la matérialité hybride des discours en contexte numérique (langagière, iconique, hypertextuelle...) (p. 269). La richesse et la diversité des exemples étudiés et des illustrations constituent des pistes méthodologiques intéressantes pour celles et ceux qui souhaitent prêter attention à la dimension composite, multimodale et intertextuelle des données numériques. Le chapitre 9 (Julien Longhi) examine les propriétés linguistiques d'un corpus de 34273 tweets politiques, constitué le 27 mars 2014, à partir de comptes Twitter de 7 personnalités issues de 6 groupes politiques ${ }^{7}$. L'hypothèse retenue porte sur la présence de corrélations entre le statut énonciatif des tweets et l'information qu'ils véhiculent, en d'autres termes sur les liens entre des traits linguistiques et leurs contenus. L'approche articule une analyse quantitative (outil de statistique textuelle, Iramuteq) à une analyse qualitative (analyse « manuelle », p. 297). Trois sous-corpus ont été constitués. Un corpus de « tweets idéologiques », identifiés à partir de marques linguistiques (absence de deixis spatiotemporels, de sujets d'interlocution etc. p. 296) qui a permis d'identifier des thématiques qualifiées d'idéologiques comme l'éducation, l'énergie... (p. 303). Un corpus de « tweets efficaces » car ayant été les plus retweetés qui concernent principalement Nicolas Sarkozy en contexte électoral, le Front National et les débats autour du mariage pour tous (p. 305). Un corpus de « tweets négatifs », contenant une forme de négation, qui met au jour la présence de thématiques différentes : pacte de responsabilité et l'euro, le débat Sarkozy/Taubira/Hollande, l'élection municipale de 2014 et la question des impôts (p. 312). La prise en compte de la matérialité linguistique des tweets est un des points forts de l'ouvrage, comme l'illustre ce chapitre. Julien Longhi y souligne en conclusion l'intérêt d'avoir recours à une approche lexicale reposant sur des méthodes fréquentielles et statistiques pour observer en quoi la mise en mots des tweets contribue à véhiculer certaines thématiques et favorise le partage d'information.

Le dernier chapitre s'intéresse au rôle des émoticônes (Chapitre 10, Arnaud Mercier) et rend compte de la diversité des données analysées et de la variété 
des approches. Les émoticônes contribuent au désir de donner de la visibilité aux tweets avec la forte présence du symbole du haut-parleur (p. 324). Ils permettent également d'exprimer des émotions (colère, horreur...) (p. 326) ou de juger l'actualité, ses acteurs et/ou les journalistes (p. 329). L'auteur souligne l'importance des émoticônes dans le cas de postures humoristiques ou ironiques (p. 330), ainsi que l'usage hyperbolique des émoticônes (p. 333). Ce chapitre illustre la nécessité de prêter attention à la variété des signes en contexte numérique pour mettre au jour leur portée argumentative : ces pictogrammes sont un élément clef de la culture Internet et contribuent à l'interprétation des messages.

De nombreuses observations ne sont pas exclusives à Twitter ou Facebook et attestent de la circulation des graphies du web et de la dimension technolangagière des productions en contexte numérique. Contrairement aux idées reçues, nourries par l'engouement pour les big data, les auteurs soulignent dès l'introduction que la collecte des données a nécessité un temps long et fastidieux de préparation et de nettoyage et s'est avérée d'une « complexité insoupçonnée » (p. 14). Ainsi, indépendamment de la sous-traitance de cette étape cruciale à une entreprise spécialisée pour la collecte des tweets, l'ouvrage met à mal la croyance d'une apparente facilité de collecte et de traitement des données en contexte numérique. Il a ainsi fallu de nombreux mois et l'aide de plusieurs chercheurs pour nettoyer et préparer les données. La diversité des méthodes (quantitatives et qualitatives) et des approches s'avère précieuse car elle contribue à nourrir les réflexions méthodologies inhérentes aux recherches sur le numérique. La diversité des protocoles et des aspects étudiés permet d'apprécier la robustesse des résultats produits, notamment quant aux caractéristiques sémio-discursives des données et de leur multimodalité (rôle des images, photomontages, émoticônes...). Certains chapitres, comme les chapitres 5 et 6 mettent en relief le pluralisme relatif du partage d'information. Comme le souligne la conclusion du chapitre 1, de nombreuses pistes de travail se dessinent, en particulier au regard d'une perspective plus sociologique qui permettrait de mieux apprécier les profils des partageurs d'information sur Twitter et Facebook, tout autant que le profil de celles et ceux qui paramètrent les bots ou newsbots. En effet, si les méthodes et démarches adoptées dans l'ouvrage présentent des « façons de faire » pour analyser les productions en ligne, en particulier du point de vue des sciences du langage et des sciences de l'information et de la communication, une perspective plus sociologique compléterait utilement les résultats. Cette 
recherche rappelle ainsi l'importance et la place des SHS pour objectiver les transformations en cours, de même que l'intérêt d'articuler des approches quantitatives et qualitatives, issues de différentes disciplines. 